Original Research Paper

\title{
The 'Umbrella' Function Describing the Density of External Electrons Injected in NaCl Crystal
}

\author{
${ }^{1}$ KAMP, Departemen Fisika, FMIPA, Universitas Gadjah Mada, Yogyakarta, Indonesia \\ ${ }^{2}$ Jurusan Fisika, FMIPA, Universitas Negeri Semarang, Indonesia \\ ${ }^{3}$ Jurusan Pendidikan Fisika, FMIPA, Universitas Negeri Yogyakarta, Indonesia
}

${ }^{1,2}$ Siti Wahyuni, ${ }^{1}$ Akrom Khasani, ${ }^{3}$ Wipsar Sunu Brams Dwandaru and ${ }^{1}$ Muhammad Farchani Rosyid

Article history

Received: 27-04-2016

Revised: 30-11-2016

Accepted: 01-12-2016

Corresponding Author: Muhammad Farchani Rosyid KAMP, Departemen Fisika, FMIPA, Universitas Gadjah Mada, Yogyakarta, Indonesia Email: farchani@ugm.ac.id

\begin{abstract}
Electrons injected in $\mathrm{NaCl}$ crystal are studied using ThomasFermi-Dirac-von Weizsacker (TFDW) model. The external potential that contributes to the TFDW energy functional is not only due to the nuclei but also to the internal electrons. In order to obtain the potential term due to the internal electrons, the density of the internal electrons in the crystal is derived from the Fourier transformation of the structure factor equation. The external potential in the TFDW energy functional is equal to the summation of the potential owing to the internal electrons and the potential due to the nuclei. The external potential determines the 'umbrella' function that bounds the minimizer of the TFDW energy functional from above. In this present work, we assume that the density of the internal electrons is not affected by the injected external electrons.
\end{abstract}

Keywords: TFDW, Internal Electron, Structure Factor, Umbrella Function, Minimizer

\section{Introduction}

TFDW functional is an extension of the simpler model of Thomas-Fermi theory, which is considered as the precursor for the birth of density functional theory. TFDW theory has been studied intensively for instance in Chen et al. (2016) where they show the existence results for the periodic TFDW equations. Furthermore, $\mathrm{Lu}$ et al. (2016) uses the orbital-free density functional theory of TFDW type to explain the response of a single layer graphene sheet on a dielectric substrate due to a point charge or collective charges.

The TFDW energy functional is given by:

$$
\begin{aligned}
E[\rho] & =\frac{3}{5}\left(3 \pi^{2}\right)^{2 / 3} \frac{\hbar^{2}}{2 m} \int \rho^{5 / 3}(x) d x+\frac{\hbar^{2}}{2 m} \int\left|\left(\nabla \rho^{1 / 2}(x)\right)\right|^{2} d x \\
& -\frac{1}{4 \pi^{3}}\left(3 \pi^{2}\right)^{4 / 3} \int \rho^{4 / 3}(x) d x+\frac{1}{2} \iint \frac{\rho(x) \rho(y)}{|x-y|} d x d y \\
& -\int V(x) \rho(x) d x
\end{aligned}
$$

where, $V(x)$ is the external potential influencing the electron gas under consideration. Lu and Otto (2014) studied the TFDW model by excluding the external potential. The result of this mathematical analysis produced the estimation of the minimizer in the interval 0 and $(4 / 5)^{3}$. They also showed that this functional has no minimizer when the number of electrons exceeds a certain positive integer. This nonexistence problem arises when we consider the small volume fraction limit for electrons in a box with uniform background charges, or physically known as the Jellium model.

Wahyuni et al. (2014) studied the TFDW model by taking into account the external potentials. In the work the authors introduced the so-called umbrella function $b^{2}(x)$. The function serves as an upper bound the minimizer of the TFDW functional, so that the minimizer assumes its values between 0 and the value of the umbrella function $b^{2}(x)$. This function is given by:

$b^{2}(x)=\left(\frac{4+\sqrt{16-60 V(x)}}{10}\right)^{3}$

where, $V(x)$ is the external potential.

Salt, also known in scientific literature as sodium chloride $(\mathrm{NaCl})$ is an ionic compound which is important in de-icing (Huber et al., 2016; Alava et al., 2016; Farnam et al., 2016) and producing various chemicals (Tan et al., 2016; Kurihara et al., 2016). Here, the $\mathrm{NaCl}$ crystal is further observed by studying the umbrella function of electrons injected into $\mathrm{NaCl}$ crystal via the TFDW model. Perhaps, the study is in spirit corresponds to another study conducted by Fujita et al. 
(2016) where electron beam is irradiated into alkalihalide nanocrsytals, e.g., $\mathrm{KCl}$ and $\mathrm{NaCl}$, to form thin graphitic carbon cages.

The present work is in the sequel of Wahyuni et al. (2014). Now we take into account the potential due to all protons of the atoms of the crystal as well as the potential due to all internal electrons of the atoms of the crystal. Therefore, the internal electrons density of the crystal should be calculated in order to determine their contribution to the total external potential in the TFDW energy functional. The density of the internal electrons in the crystal is determined from the Fourier transformation of the structure factor equation. Furthermore, the internal electron density of the crystal, the potential due to the internal electron density, the potential due to all protons of the nuclei, the total external potential and the yielded umbrella function estimating the minimizer of the TFDW energy functional are sketched.

\section{The 'Umbrella' Function of TFDW Model}

The TFDW energy functional for electrons with external potential $V$ is given by:

$$
E[\phi]:=\int\left[|\nabla \phi|^{2}+F\left(\phi^{2}\right)\right] d x+D\left(\phi^{2}, \phi^{2}\right)+\int V \phi^{2} d x
$$

where, $F(t)=t^{5 / 3}-t^{4 / 3}$ and $D(\cdot, \cdot)$ is the Coulomb interaction in $R^{3}$ i.e.:

$$
D(f, g)=\iint \frac{f(x) g(y)}{|x-y|} d x d y
$$

Equation 1 can be rewritten as:

$$
E[\phi]:=\int\left[|\nabla \phi|^{2}+\bar{F}\left(\phi^{2}\right)\right] d x+D\left(\phi^{2}, \phi^{2}\right)
$$

where, $F(t)=t^{5 / 3}-t^{4 / 3}+V t$.

The variational principle related to the TFDW model is to find the minimizer of the TFDW functional energy, i.e., a function $\phi$ that minimizes the functional $E[\varphi]$ in the sense:

$$
E[\varphi]=\inf \inf _{\phi \in X, \int \phi^{2}=m} E[\phi]
$$

for suitable function space $X$.

From the main result stated in Wahyuni et al. (2014), the value of minimize $\phi(x)$ is always lower than the value of the function $b^{2}(x)$ for every $x$ in a suitable domain, so that this function serves as an upper bound of the minimizer. This is the reason that the function $b^{2}(x)$ is called the 'umbrella' function.

\section{The Internal Electron Density of $\mathrm{NaCl}$}

An image of the crystal structure can be calculated from the X-ray diffraction pattern. The pattern reflects the electron density at every point in the unit cell of the crystal. The electron density is expressed as:

$\rho(x y z)=\frac{1}{V} \sum_{h k l} F(h k l) \exp [-2 \pi i(h x+k y+l z)]$

where, the summation is over all structure factor $F(h k l)$ and $V$ is the volume of unit cell. The structure factor is the only factor that determines the X-ray intensity on a diffraction pattern that depends on crystal structures. This factor can be stated in terms of the contents of a single unit cell as:

$$
F(h k l)=\sum_{j=1}^{N} f_{j} e^{2 \pi i\left(h x_{j}+k y_{j}+l z_{j}\right)}
$$

where, $\left(x_{j}, y_{j}, z_{j}\right)$ is the $j$-th atom position, $f_{j}$ is its scattering factor and $N$ is the number of atoms in the cell. The structure factor is calculated in term of the number of electrons. It gives a mathematical description of the diffraction pattern. Each structure factor represents a diffracted beam with an amplitude $|F(h k l)|$ and relative phase $\phi(h k l)$. Further study can be found in Blake et al. (2009; Szwacki and Szwacka, 2010).

Sodium chloride $(\mathrm{NaCl})$ has a face centered cubic crystal lattice. Based on the JCPDS card with the file number of 5-0628 from the database, we obtain information that the edge $a$ is $5.6402 \AA$ (Waseda et al., 2011). An illustration of $\mathrm{NaCl}$ structure is depicted in Fig. 1. The solid (blue and red) dots are the $\mathrm{Na}$ and $\mathrm{Cl}$ atoms. The distance between two adjacent red or blue dots is $a$.

We find the $\mathrm{NaCl}^{\mathrm{s}}$ structure factor as:

$$
F(h k l)=\sum_{j=1}^{N} f_{N a^{+}} e^{2 \pi i\left(h x_{j}+k y_{j}+l z_{j}\right)}+\sum_{j=1}^{N} f_{C l^{-}} e^{2 \pi i\left(h x_{j}+k y_{j}+l z_{j}\right)}
$$

For $\mathrm{Na}^{+}$with ions positions $\left(\begin{array}{lll}0 & 0 & 0\end{array}\right),\left(\begin{array}{lll}0 & 1 / 2 & 1 / 2\end{array}\right),\left(\begin{array}{lll}1 / 2 & 0\end{array}\right.$ $1 / 2)$ and (1/2 1/2 0), we get:

$e^{2 \pi i\left(h x_{j}+k y_{j}+l z_{j}\right)}=1+e^{\pi i(k+l)}+e^{\pi i(h+l)}+e^{\pi i(h+k)}$

while for $\mathrm{Cl}^{-}$with ions positions $\left(\begin{array}{lll}1 / 2 & 0 & 0\end{array}\right),\left(\begin{array}{lll}0 & 1 / 2 & 0\end{array}\right),\left(\begin{array}{ll}0 & 0\end{array}\right.$ $1 / 2)$ and (1/2 1/2 1/2), we get:

$e^{2 \pi i\left(h x_{j}+k y_{j}+l z_{j}\right)}=e^{\pi i h}+e^{\pi i k}+e^{\pi i(h+k+l)}$

so that:

$$
\begin{aligned}
F(h k l)= & f_{N a^{+}}\left[1+e^{\pi i(k+l)}+e^{\pi i(h+l)}+e^{\pi i(h+k)}\right]+ \\
& f_{C l^{-}} e^{\pi i(h+k+l)}\left[e^{-\pi i(k+l)}+e^{-\pi i(h+l)}+e^{-\pi i(h+k)}+1\right]
\end{aligned}
$$




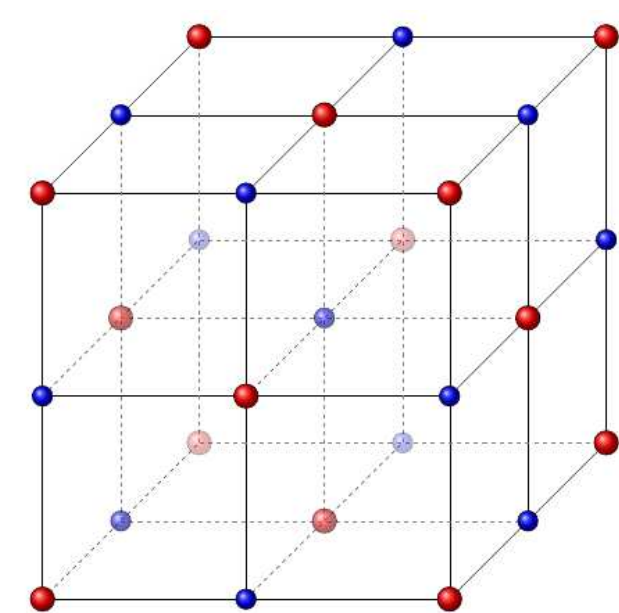

Fig. 1. The structure of $\mathrm{NaCl}$

Recalling that if $n$ is any integer then $e^{n \pi i}=e^{-n \pi i}$, we can rewrite Equation 12 as:

$$
\begin{aligned}
F(h k l)= & \left(f_{\mathrm{Na}^{+}}+f_{C l^{-}} e^{\pi i(h+k+l)}\right) \\
& \left(1+e^{\pi i(k+l)}+e^{\pi i(h+l)}+e^{\pi i(h+k)}\right)
\end{aligned}
$$

From Equation 13 we conclude that:

- When $h, k, l$ are even, then $F(h k l)=4\left(f_{N a^{+}}+f_{C l-}\right) \rightarrow$ primary reflections

- When $h, k, l$ are odd, then $F(h k l)=4\left(f_{\mathrm{Na}+}-f_{\mathrm{Cl}}\right) \rightarrow$ superlattice reflections

- When $h, k, l$ are mixed, then $F(h k l)=0 \rightarrow$ no reflections

The atomic scattering factors $f_{\mathrm{Na}+}$ and $f_{\mathrm{Cl}}$ are calculated using the Bragg law:

$$
\lambda=2 d \sin \theta
$$

where, $d$ for cubic crystal is:

$$
d_{h k l}=\frac{a}{\sqrt{h^{2}+k^{2}+l^{2}}}
$$

as well as the appropriate data in the International Tables for Crystallography (Prince, 2004).

\section{Material and Methods}

In principle, this is a theoretical and numerical study. For numerical calculation we use Matlab 2015. The physical system under consideration is electrons which are injected into $\mathrm{NaCl}$ crystal. The study is based upon the TFDW model where the umbrella function of the aforementioned system is to be determined. The contributions to the external potential are from all of the internal electrons and protons of the $\mathrm{NaCl}$ crystal.

From Equation 8, in the calculation of the structure factor, we take all possible Miller indices into account, so we should know the Miller planes producing the intensity of the diffraction pattern. To that end, we make use of the experimental data of $\mathrm{NaCl}$ diffraction. If we take the information from the JCPDS card of the file number 5-0628 from the database, we know that the Miller planes raising the intensity of the diffraction pattern is of 17 planes, all of which contribute to the structure factor calculation. Furthermore, from the Miller indices data, the scattering factor of $\mathrm{Na}^{+}$and $\mathrm{Cl}^{-}$are calculated appropriately with the result of $\sin \theta / \lambda$ according to Bragg law formula. To find the appropriate scattering factor, it is sufficient to use the data from the International Table for Crystallography, appropriate with the calculation result and then take an interpolation. The scattering factor that has been obtained is used to calculate the structure factor, i.e., using Equation 12.

Once the structure factor has been calculated, the density of all of the internal electrons of the $\mathrm{NaCl}$ crystal may be obtained and hence the external potential due to all of the internal electrons. Add the latter to the potential contribution of all protons in the crystal we finally obtain the total external potential. Hence, the umbrella function of the electrons injected into the $\mathrm{NaCl}$ crystal may be gained.

\section{Results}

The results of the calculation of the scattering factor and structure factor of the $\mathrm{Na}^{+}$and $\mathrm{Cl}^{-}$are presented in Table 1 . The structure factors $F(h k l)$ obtained in the first step are used in turn for calculating the internal electron density of $\mathrm{NaCl}$, i.e., by using Equation 7. Then the result of the calculation can be written separately into two terms, i.e., the real part and the imaginary part. Overall, the calculation of the internal electron density is presented in Table 2; however, it is still expressed in terms of Miller's plane.

The magnitude of the density is equal to the summation of the modulus of the 17 terms of the Miller's planes. Then, the distribution of the internal electron density can be calculated and sketched. The obtained density of the internal electrons gives the potential term due to the internal electrons and together with that of the protons of all nuclei contributes to the total external potential term in the TFDW energy functional.

\section{The Potential Due to the Internal Electron of the Crystal}

As we have mentioned above, the density of the internal electrons of the crystal is calculated in order to determine the potential term due to the internal electrons. 
Table 1. The calculation of scattering factor and structure factor of the $\mathrm{Na}^{+}$and $\mathrm{Cl}^{-}$

\begin{tabular}{llllllll}
\hline$H$ & $k$ & $l$ & $d_{h k l}$ & $\sin \theta / \lambda$ & $f_{N a^{+}}$ & $f_{C l^{-}}$ & $F_{h k l}$ \\
\hline 1 & 1 & 1 & 3.256 & 0.154 & 8.983 & 13.614 & -18.524 \\
2 & 0 & 0 & 2.820 & 0.177 & 8.682 & 12.748 & 85,722 \\
2 & 2 & 0 & 1.994 & 0.251 & 7.635 & 10.563 & 72.790 \\
3 & 1 & 1 & 1.701 & 0.294 & 6.984 & 9.639 & -10.619 \\
2 & 2 & 2 & 1.628 & 0.307 & 6.789 & 9.403 & 64.769 \\
4 & 0 & 0 & 1.410 & 0.355 & 6.095 & 8.686 & 59.124 \\
3 & 3 & 1 & 1.294 & 0.386 & 5.653 & 8.308 & -10.620 \\
4 & 2 & 0 & 1.261 & 0.396 & 5.519 & 8.200 & 54.875 \\
4 & 2 & 2 & 1.151 & 0.434 & 5.035 & 7.836 & 51.485 \\
5 & 1 & 1 & 1.085 & 0.461 & 4.721 & 7.611 & -11.560 \\
4 & 4 & 0 & 0.997 & 0.501 & 4.276 & 7.294 & 46.281 \\
5 & 3 & 1 & 0.953 & 0.524 & 4.054 & 7.129 & -12.299 \\
6 & 0 & 0 & 0.940 & 0.532 & 3.983 & 7.075 & 44.231 \\
6 & 2 & 0 & 0.892 & 0.561 & 3.720 & 6.871 & 42.365 \\
5 & 3 & 3 & 0.860 & 0.581 & 3.549 & 6.729 & -12.718 \\
6 & 2 & 2 & 0.850 & 0.588 & 3.494 & 6.683 & 40.706 \\
4 & 4 & 4 & 0.814 & 0.614 & 3.296 & 6.503 & 39.197 \\
\hline
\end{tabular}

Table 2. The component of the internal electron density

\begin{tabular}{lll}
\hline$h k l$ & Real parts & Imaginary parts \\
\hline$(111)$ & $-18.524 \cos 2 \pi(x+y+z)$ & $18.524 \sin 2 \pi(x+y+z)$ \\
$(200)$ & $85.722 \cos 4 \pi x$ & $-85.722 \sin 4 \pi x$ \\
$(220)$ & $72.790 \cos 4 \pi(x+y)$ & $-72.790 \sin 4 \pi(x+y)$ \\
$(311)$ & $-10.619 \cos 2 \pi(3 x+y+z)$ & $10.619 \sin 2 \pi(3 x+y+z)$ \\
$(222)$ & $64.769 \cos 4 \pi(x+y+z)$ & $-64.769 \sin 4 \pi(x+y+z)$ \\
$(400)$ & $59.124 \cos 8 \pi x$ & $-59.124 \sin 8 \pi x$ \\
$(331)$ & $-10.620 \cos 2 \pi(3 x+3 y+z)$ & $10.620 \sin 2 \pi(3 x+3 y+z)$ \\
$(420)$ & $54.875 \cos 4 \pi(2 x+y)$ & $-54.875 \sin 4 \pi(2 x+y)$ \\
$(422)$ & $51.485 \cos 4 \pi(2 x+y+z)$ & $-51.485 \sin 4 \pi(2 x+y+z)$ \\
$(511)$ & $-11.560 \cos 2 \pi(5 x+y+z)$ & $11.560 \sin 2 \pi(5 x+y+z)$ \\
$(440)$ & $46.281 \cos 8 \pi(x+y)$ & $-46.281 \sin 8 \pi(x+y)$ \\
$(531)$ & $-12.299 \cos 2 \pi(5 x+3 y+z)$ & $12.299 \sin 2 \pi(5 x+3 y+z)$ \\
$(600)$ & $44.231 \cos 12 \pi x$ & $-44.231 \sin 12 \pi x$ \\
$(620)$ & $42.365 \cos 4 \pi(3 x+y)$ & $-42.365 \sin 4 \pi(3 x+y)$ \\
$(533)$ & $-12.718 \cos 2 \pi(5 x+3 y+3 z)$ & $12.718 \sin 2 \pi(5 x+3 y+3 z)$ \\
$(622)$ & $40.706 \cos 6 \pi(2 x+y+z)$ & $-40.706 \sin 6 \pi(2 x+y+z)$ \\
$(444)$ & $39.197 \cos 8 \pi(x+y+z)$ & $-39.197 \sin 8 \pi(x+y+z)$ \\
\hline
\end{tabular}

The potential term together with that of the protons of all nuclei contributes to the total external potential term in the TFDW energy functional. The potential term due to the internal electrons of the crystal is given by:

$$
V_{i}(x)=\int \frac{\rho_{i}(y)}{|x-y|} d y
$$

where, $\rho_{i}$ is the internal electron density. The total external potential $V(x)$ of the TFDW energy functional is given by:

$$
V(x)=\sum_{m \in Z^{3}} \sum_{j=0}^{n-1} q_{j}\left|A m+d_{j}-x\right|^{-1}+\int \frac{\rho_{i}(y)}{|x-y|} d y
$$

where, $A, m$ and $d_{j}$ are the primitive unit cell matrix, site and position vector of the any point $\boldsymbol{x}$ to the proton $q_{j}$, respectively.

\section{Discussion}

It is shown how the presence of the potential term due to all protons of all nuclei and the potential term due to the internal electrons of the crystal shapes the associated umbrella function. The change in the external potential, of course, affects the umbrella function. The computer sketch of the internal electron density of the crystal, the potential due to the internal electron density, the potential due to all protons of the nuclei, the total external potential and the umbrella function estimating the minimizer of the TFDW energy functional are depicted respectively in Fig. 2 from the left to the right for various slicing of $z$-axis. 

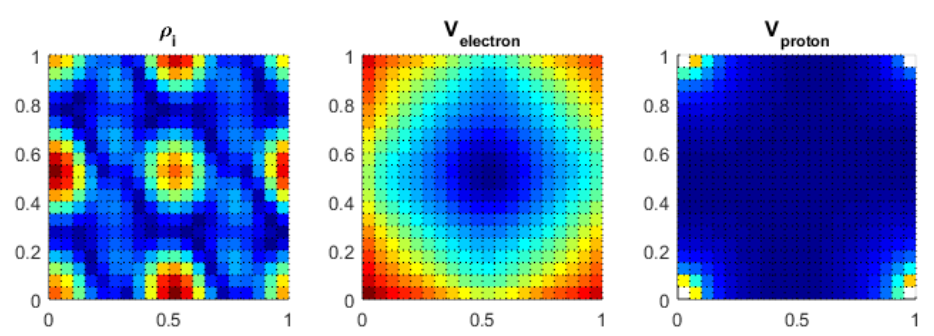

(a)
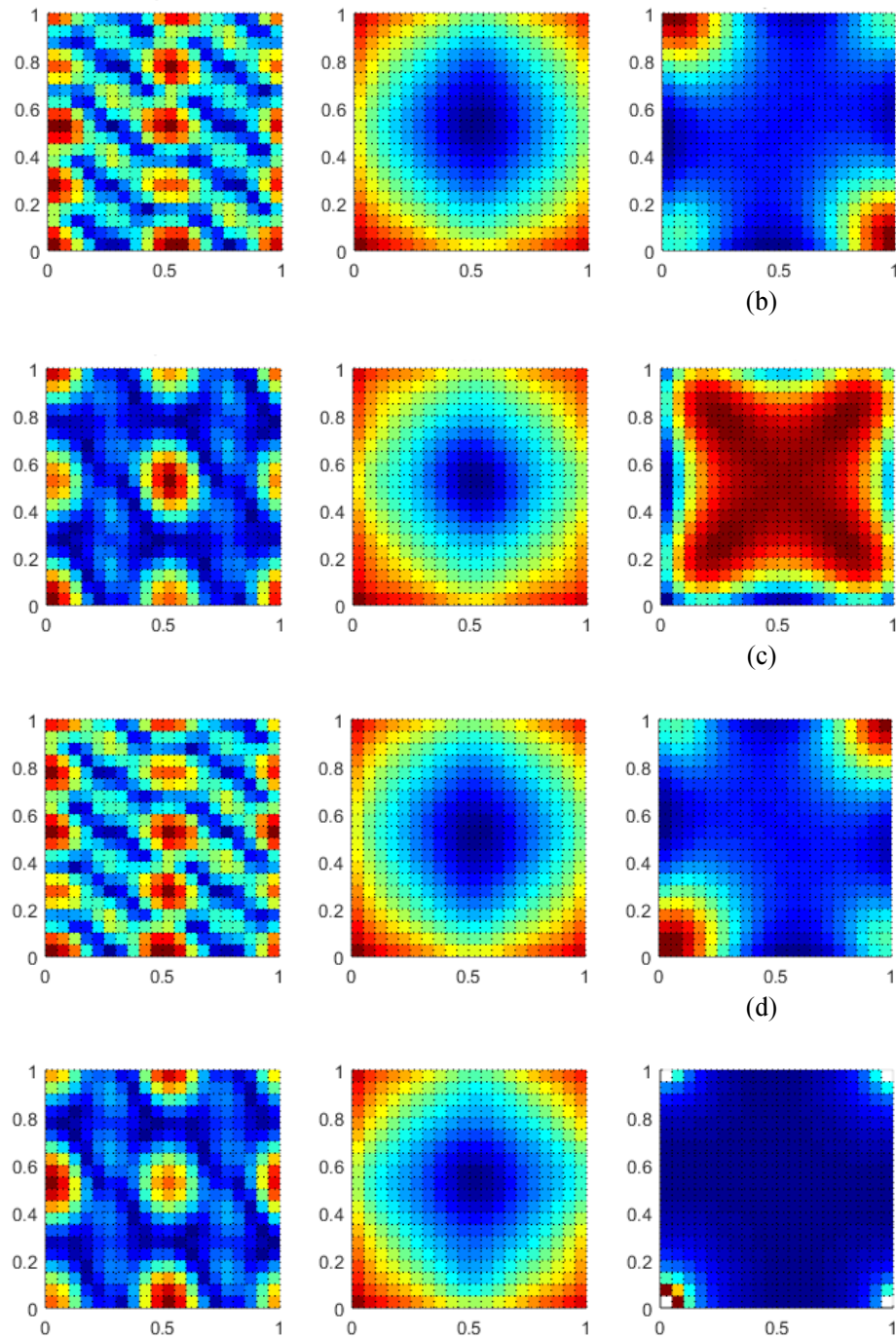

(b)

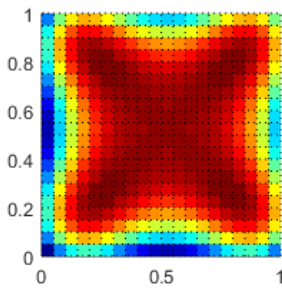

(c)

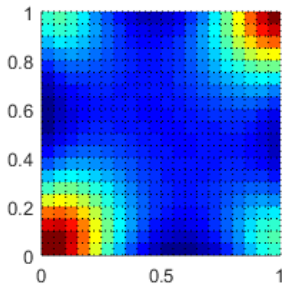

(d)

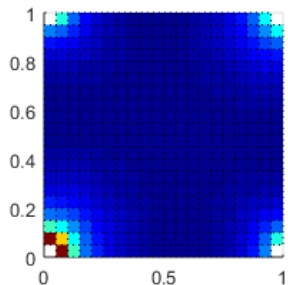

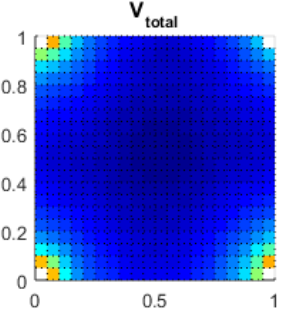
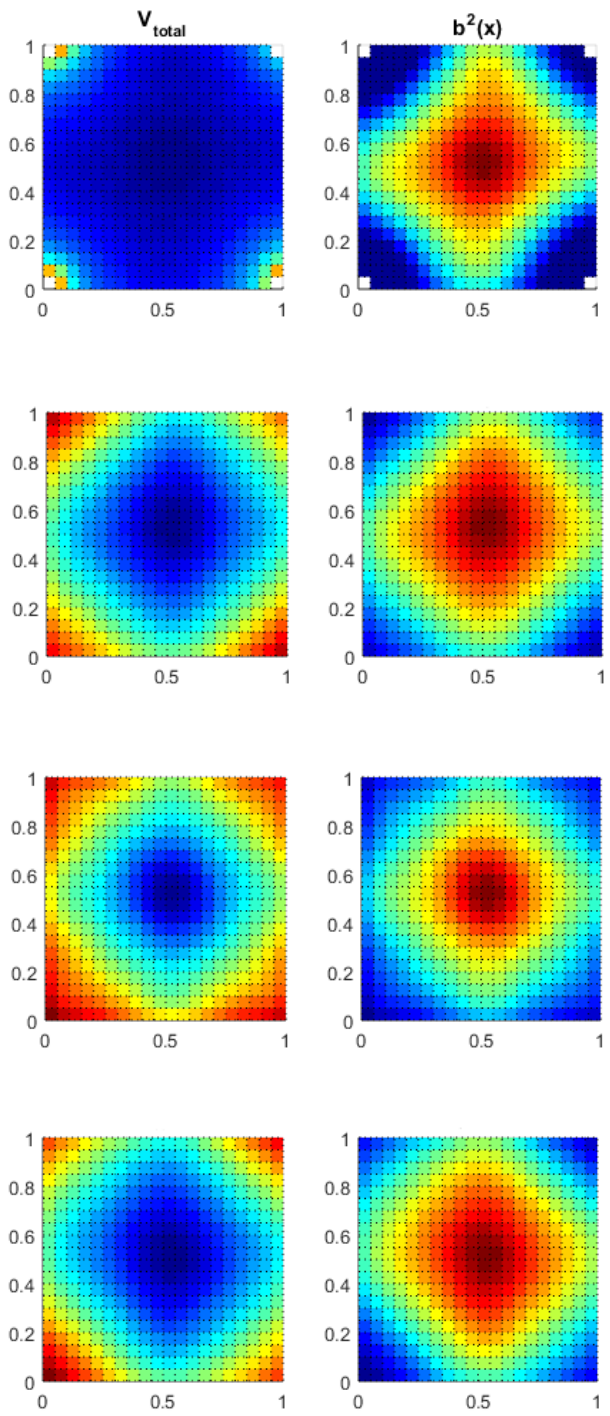

(e)
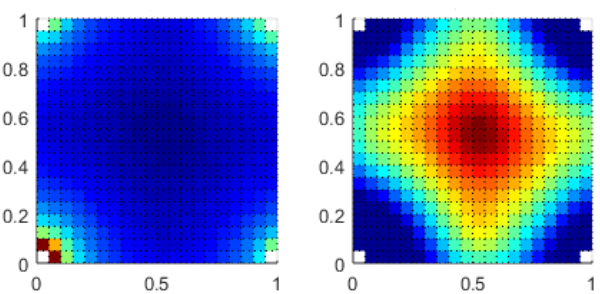

Fig. 2. The density of internal electrons, the potential of internal electrons, the potential of proton, the total potential and the umbrella function on several slicing (a) $z=0$, (b) $z=0.25$, (c) $z=0.5$, (d) $z=0.75$, (e) $z=1$

Observe Fig. 2 on the first line, i.e., the $z=0$ slicing. The red color on the sketch of the internal electron density indicates the location most occupied by the internal electrons. On the fourth sketch, i.e., the sketch of the total external potential due to all protons of the nuclei and the internal electrons, blue color spread on almost all areas of the plane, except in the area around the corner points. This situation is shown more clearly on the leftmost picture on the upper line of Fig. 3, where the contour of the total potential is given. The potential is negative on the central region and positive around the corner points. This means that if there is an external electron entering the crystal, the electron will be rejected by the regions around the corner points. 
(a) $\mathrm{v}$

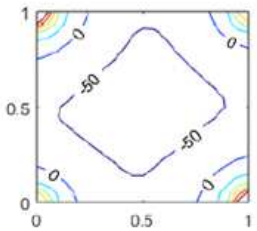

(b) $b^{2}(x)$

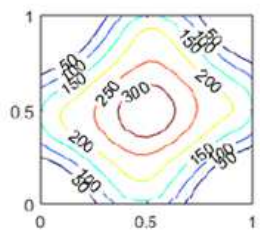

$z=0$
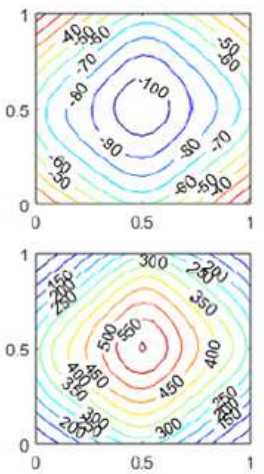

$z=0.25$
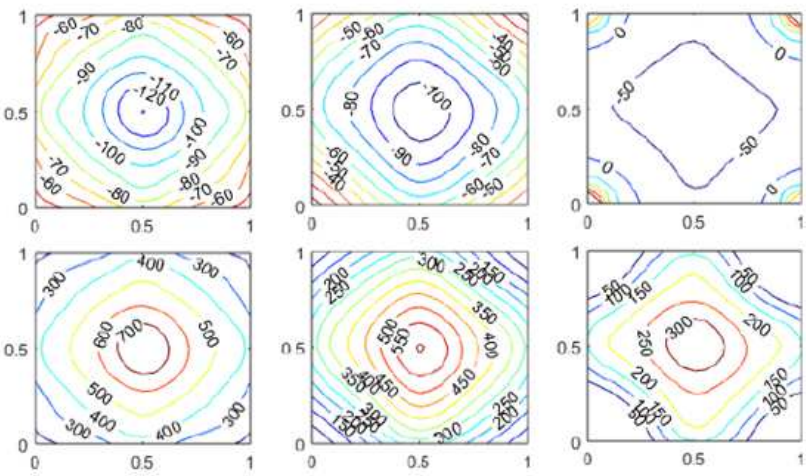

$z=0.5$

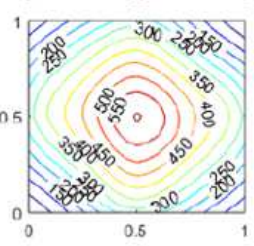

$z=0.75$

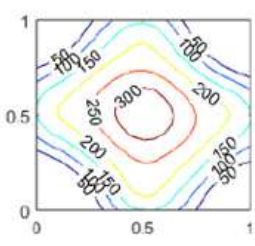

$z=1$

Fig. 3. The contour of (a) the total potential on various slicing and (b) the umbrella function on various slicing

Thus, external electrons tend to occupy the central region or the blue area. The central region of the plane will be the most occupied region by the external electrons entering the crystal. Such behavior is also predicted consistently by the umbrella function on the slicing, the sketch of which is given on the rightmost picture of the first line of Fig. 2 or on the leftmost picture on the lower line of Fig. 3. The sketch of the umbrella function reflects the minimizer of the TFDW energy functional on $z=0$ slicing. The red colored regions on the plane of the sketch of the umbrella function represent the most possibly occupied region by external electrons. The sketches on the other slicing can be interpreted in similar ways.

Now observe the contours of the umbrella function shown on the Fig. 3a, i.e., on the lower line of Fig. 3. The external electrons on the slicing $z=0$ and $z=1$ will be most localized, while the external electrons on the slicing $z=0.25$ and $z=0.75$ will be less localized than that on the slicing $z=0$ and $z=1$. The external electrons on the central slicing $(z=0.5)$ will be more spread. This means that the external electrons injected in the $\mathrm{NaCl}$ tend to occupy the middle region of the crystal. This is because of low probability of internal electrons being in the middle region of the crystal.

\section{Conclusion}

The behavior of the external electrons injected in $\mathrm{NaCl}$ crystal predicted by the umbrella function is consistent with the total external potential which is equal to the summation of the potential terms caused by the internal electrons and the potential term due to the protons of all nuclei of the crystal. The umbrella function associated with the total external potential can be used for estimating the minimizer of the TFDW energy functional.

\section{Acknowledgment}

We would like to thank Bambang Sugeng, M. Pd., $\mathrm{Ph} . \mathrm{D}$. for reviewing the language in the manuscript.

\section{Author's Contributions}

S. Wahyuni is the main researcher of project. She conducted the theoretical calculation and providing the initial draft of the manuscript. A. Khasani contributed in the computational calculation and setting the Matlab program in the research. W.S.B. Dwandaru is the second supervisor (co-promotor) for S. Wahyuni and oversaw the theoretical calculation and review the content of the manuscript. Finally, M.F. Rosyid is the first supervisor (promotor) for S. Wahyuni who provided the main idea and oversaw the overall research project as well as reviewing the manuscript.

\section{Ethics}

This article is original and to the best knowledge of the authors has not been published before. The authors confirm that there are no ethical issues involved.

\section{References}

Alava, H.E., N. De Belie and G. De Schutter, 2016. Proposed mechanism for the formation of oxychloride crystals during sodium chloride application as a deicer salt in carbonated concrete. Construct. Build. Mater., 109: 188-197. DOI: 10.1016/j.conbuildmat.2016.01.047

Blake, A.J., W. Clegg, J.M. Cole, J.S.O. Evans and P. Main et al., 2009. Crystal Structure Analysis: Principles and Practice. 1st Edn., Oxford University Press, Oxford, ISBN-10: 019921946X, pp: 387.

Chen, S., L. Lin and L. Xiao, 2016. Existence Results for the periodic thomas-fermi-dirac-von weizsäcker equations. Adv. Math. Phys. DOI: $10.1155 / 2015 / 652407$

Farnam, Y., C. Villani, T. Washington, M. Spence and J. Jain et al., 2016. Performance of carbonated calcium silicate based cement pastes and mortars exposed to $\mathrm{NaCl}$ and $\mathrm{MgCl}_{2}$ deicing salt. Construct. Build. Mater., 111: 63-71.

DOI: 10.1016/j.conbuildmat.2016.02.098 
Fujita, J., M. Tachi, N. Ito, K. Murakami and M. Takeguchi, 2016. Graphitic cage transformation by electron-beam-induced catalysis with alkalihalide nanocrystals. Jap. J. Applied Phys., 55: 055102-055102. DOI: 10.7567/JJAP.55.055102

Huber, M., H. Hilbig, S.C. Badenberg, J. Fassnacht and J.E. Drewes et al., 2016. Heavy metal removal mechanisms of sorptive filter materials for road runoff treatment and remobilization under de-icing salt applications. Water Res., 102: 453-463.

DOI: $10.1016 /$ j.watres.2016.06.063

Kurihara, M., F. Hayashi, K. Shimizu, H. Wagata and T. Hirano et al., 2016. Flux-boosted sulfide crystal growth: Growth of $\mathrm{CuInS}_{2}$ crystals by $\mathrm{NaCl}-\mathrm{InCl}_{3}$ evaporation. Cryst. Growth Des., 16: 1195-1199. DOI: $10.1021 /$ acs.cgd.5b01142

Lu, J. and F. Otto, 2014. Nonexistence of a minimizer for thomas-fermi-dirac-von weizsäcker model. Commun. Pure Applied Math., 67: 1605-1617. DOI: $10.1002 /$ cpa.21477

Lu, J., V. Moroz and C.B. Muratov, 2016. Orbital-free density functional theory of out-of-plane charge screening in graphene. J. Nonlinear Sci., 25: 1391-1430. DOI: 10.1007/s00332-015-9259-4
Prince, E., 2004. International Tables for Crystallography, Volume C: Mathematical, Physical and Chemical Tables. 3rd Edn., Dordrecht, Springer Science and Business Media, ISBN-10: 1402019009, pp: 1000.

Szwacki, N.G. and T. Szwacka, 2010. Basic Elements of Crystallography. 1st Edn., Pan Stanford Publishing, Singapore, ISBN-10: 9814241598, pp: 195.

Tan, K.T., C.C. White, D. Hunston, J. M. Gorham and M.J. Imburgia et al., 2016. Role of salt on adhesion of an epoxy/aluminum (Oxide) interface in aqueous environments. Polymer Eng. Sci., 56: 18-26. DOI: $10.1002 /$ pen. 24186

Wahyuni, S., W.S.B. Dwandaru and M.F. Rosyid, 2104. On the minimizer of the thomas-fermi-dirac-von weizsäcker model. J. Phys. Conf. Series, 539: 1-4.

Waseda, Y., E. Matsubara and K. Shinoda, 2011. X-Ray Diffraction Crystallography: Introduction, Examples and Solved Problems. 1st Edn., Springer Science and Business Media, Berlin ISBN-10: 3642166350 , pp: 310 . 